\title{
Frenkel-Kontorova Model with Toda Interactions
}

\author{
Bin Lin \\ Department of Physics \\ Case Western Reserve University \\ Cleveland, Ohio 44106-7079 \\ Bambi Hu \\ Department of Physics \\ University of Houston \\ Houston, Texas 77024-5506
}

We have studied the Frenkel-Kontorova (FK) model with Toda interactions. The phase diagram is found to be asymmetric due to the exponential form of the Toda interaction. The reflectional symmetry observed in the standard FK model is broken under Toda interactions. The singularity spectrum and the generalized dimension are calculated and their dependence on the nonlinearity parameter is discussed. The critical exponents of the gap in the phonon spectrum, the correlation length and the Peierls-Nabarro barrier are calculated and found to be independent of the nonlinearity parameter, and are the same as those in the standard FK model. The FK model with Toda interactions thus seems to belong to the same universality class as the standard FK model. 


\section{INTRODUCTION}

Commensurate-incommensurate phase transitions have been observed in spinand charge-density waves, ${ }^{1,2}$ magnetic spirals, ${ }^{3}$ intercalation componnds ${ }^{4}$ and quasi-crystals. ${ }^{5}$ The Frenkel-Kontorova (FK) model $^{6}$ has been widely used to study such transitious. It is also used as a model for crystal dislocation ${ }^{\top}$ and absorbed epitaxial monolayers. ${ }^{8}$ This simple one-dimensional model has been proven to be most suitable for the description of various phenomena arising from competing periodicities. The Hamiltonian associated with the FK model may be written as

$$
\mathcal{H}=\sum_{i}\left[\frac{1}{2}\left(x_{i+1}-x_{i}-\mu\right)^{2}+\frac{k}{(2 \pi)^{2}}\left(1-\cos 2 \pi x_{i}\right)\right],
$$

where $x_{i}$ denotes the position of the $i$ th atom and $\mu$ the length of the nuperturbbed spring. The second term is the external potential with strength $k$ and period one.

In the standard FK model (1), the interatomic forces are harmonic. While interactions in some systems can be well approximated by a harmonic forces, there are many systems in which the interactions are far from being harmonic. In this paper we will study a generalized FK model with anharmonic Toda interactions. ${ }^{9,10}$ The Toda interaction $W_{0}\left(x_{i+1}-x_{i}\right)$ between two neighboring atoms is given by

$$
W_{0}\left(x_{i+1}-x_{i}\right)=\frac{1}{\beta^{2}}\left[e^{-\beta\left(x_{i+1}-x_{i}-\mu\right)}-1\right]+\frac{1}{\beta}\left(x_{i+1}-x_{i}-\mu\right),
$$

where $\beta$ is a nonlinear parameter measuring the degree of anharmonicity. When $\beta$ tends to zero. the Toda interaction reverts to the harmonic form (standard FK model); as $\beta$ increases, the interaction becomes more and more nonlinear, and in 
the limit $\beta \rightarrow \infty$, the Toda interaction becomes that of a hard-rod:

$$
\begin{array}{ll}
\beta \rightarrow 0, & W_{0}(r) \rightarrow \frac{1}{2}(r-\mu)^{2}, \\
\beta \rightarrow \infty, & W_{0}(r) \rightarrow \infty, \quad \text { if } r<\mu, \\
& W_{0}(r) \rightarrow 0, \quad \text { if } r \geq \mu,
\end{array}
$$

where $r=x_{i+1}-x_{i}$.

If we consider an external cosine potential as in the standard FK model (1), the Hamiltonian for the generalized FK model with Toda interactions (2) can be written as

$$
\mathcal{H}_{0}=\sum_{i}\left\{\frac{1}{\beta^{2}}\left[e^{-\beta\left(x_{i+1}-x_{i}-\mu\right)}-1\right]+\frac{1}{\beta}\left(x_{i+1}-x_{i}-\mu\right)+\frac{k_{0}}{(2 \pi)^{2}}\left(1-\cos 2 \pi x_{i}\right)\right\}
$$

Milchev and Mazzucchelli ${ }^{10}$ have studied the effects of the misfit $\mu$ on the soliton description of this model. They found that the soliton picture of the dislocations breaks down beyond some critical values of the misfit. The length and the density of anharmonic solitons depend in an essential way on both the sign and the magnitude of the misfit. They have also discussed the dependence of the frequency spectrum on the misfit, the nonlinear parameter $\beta$ and the external potential strength $k$.

In this paper we will concentrate on the phase diagram, the multifractal properties and the critical behaviors of some physical quantities. For these purposes, we will rewrite the Hamiltonian (4) to rearrange the parameter $\mu$. In the Hamiltonian (4), $\mu$ appears with the term $\left(x_{i+1}-x_{i}\right)$ in the exponent. This will make the boundary of phase diagram an implicit function and the mapping iteration dependent on $\mu$. Fortunately, we can rescale the Hamiltonian to avoid these difficulties. 
The new Hamiltonian $\mathcal{H}$, rescaled from $\mathcal{H}_{0}$ by a factor $e^{-\beta \mu}$, is given by

$$
\begin{aligned}
\mathcal{H} & =e^{-\beta \mu} \mathcal{H}_{0} \\
& =\sum_{i}\left\{\frac{1}{\beta^{2}}\left[e^{-\beta\left(x_{i+1}-x_{i}\right)}-e^{-\beta \mu}\right]+\frac{e^{-\beta \mu}}{\beta}\left(x_{i+1}-x_{i}-\mu\right)+\frac{k}{(2 \pi)^{2}}\left(1-\cos 2 \pi x_{i}\right)\right\}
\end{aligned}
$$

where $k=e^{-\beta \mu} k_{0}$. In the rescaled form, the interaction $W(r)=e^{-\beta \mu} W_{0}(r)$ decreases monotonically as a function of $\beta$ :

$$
\begin{array}{ll}
\beta \rightarrow 0, & W(r) \rightarrow \frac{1}{2}(r-\mu)^{2} ; \\
\beta \rightarrow \infty & W(r) \rightarrow 0 .
\end{array}
$$

Fig. 1 shows $W_{0}(r)$ and $W(r)$ for several values of $\beta$. In the following we will use the rescaled Hamiltonian (5) for all discussions. The rest of this paper is organized as follows: The ground states and the phase diagrams will be discussed in Sec. II. In Sec. III, we study the Devil's staircase, the singularity spectrum $f(\alpha)$ and the generalized dimension $D_{q}$. In Sec. IV, the critical exponents of the gap in the phonon spectrum, the correlation length and the Peierls-Nabarro barrier are computed. A brief conclusion and discussion are given in Sec. V.

\section{GROUND STATE AND PHASE DIAGRAM}

\section{A. Ground State}

Since the Toda interaction $W(r)$ is a convex function, we can use the gradient method $^{1-13}$ to calculate the periodic ground states. Consider a commensurate structure with winding number $\omega=p / q$ (the average atomic distance) which is defined by

$$
\omega=\lim _{i-i^{\prime} \rightarrow \infty} \frac{x_{i}-x_{i^{\prime}}}{i-i^{\prime}}
$$


The initial positions of the $q$ atoms are set up in an equal spacing array

$$
x_{i}(0)=i \frac{p}{q}+\alpha, \quad i=1,2, \ldots, q
$$

where the phase $\alpha$, a constant throughout the system, satisfies

$$
m_{i} \leq 2(i \omega+\alpha) \leq m_{i}+1
$$

Here $m_{i}$ is the integral part of $2 x_{i}$ :

$$
m_{i}=\left[2 x_{i}\right]
$$

Let the system evolve in time according to the $q$ differential equations

$$
\frac{d x_{i}}{d t}=f_{i}, \quad i=1,2, \cdots, q
$$

where $f_{i}$ is the force acting on $i$ th atom

$$
\begin{aligned}
f_{i} & =-\frac{\partial \mathcal{H}}{\partial x_{i}} \\
& =\frac{1}{\beta}\left[e^{-\beta\left(x_{i}-x_{i-1}\right)}-e^{-\beta\left(x_{i+1}-x_{i}\right)}\right]-\frac{k}{2 \pi} \sin 2 \pi x_{i} . \quad i=1,2, \cdots, q
\end{aligned}
$$

This evolution relaxes the system towards the ground state under the influence of the restoring forces. It has been shown ${ }^{11,13}$ that the resulting configuration is always a ground state if the initial configuration is given by (8). A periodic condition $^{14}$ must be satisfied in Eqs. (11) and (12) to ensure periodic orbits.

$$
\left\{\begin{aligned}
x_{0} & =x_{q}-p \\
x_{q+1} & =x_{1}+p .
\end{aligned}\right.
$$


The differential equations (11) can then be rewritten as

$$
\left\{\begin{aligned}
\frac{d x_{1}}{d t} & =\frac{1}{\beta}\left[e^{-\beta\left(x_{1}-x_{q}+p\right)}-e^{-\beta\left(x_{2}-x_{1}\right)}\right]-\frac{k}{2 \pi} \sin 2 \pi x_{1} \\
\frac{d x_{2}}{d t} & =\frac{1}{\beta}\left[e^{-\beta\left(x_{2}-x_{1}\right)}-e^{-\beta\left(x_{3}-x_{2}\right)}\right]-\frac{k}{2 \pi} \sin 2 \pi x_{2} \\
& \vdots \\
\frac{d x_{q-1}}{d t} & =\frac{1}{\beta}\left[e^{-\beta\left(x_{q-1}-x_{q-2}\right)}-e^{-\beta\left(x_{q}-x_{q-1}\right)}\right]-\frac{k}{2 \pi} \sin 2 \pi x_{q-1} \\
\frac{d x_{q}}{d t} & =\frac{1}{\beta}\left[e^{-\beta\left(x_{q}-x_{q-1}\right)}-e^{-\beta\left(x_{1}-x_{q-1}+p\right)}\right]-\frac{k}{2 \pi} \sin 2 \pi x_{q} .
\end{aligned}\right.
$$

These equations can be solved by the standard Runge-Kutta method. When the ground state is reached, the force on each atom should vanish. In practice, the ground state can be considered found if all $d x_{i} / d t$ become less than a given prescribed value.

This method works well, but there is a practical problem. When looking for a high period ground state, one has to integrate a large number (e.g., $q=4181$ ) of differential equations simultaneously. It takes a large amount of computer time. In fact, only the final configuration where all forces vanish is what we want. All the evolution steps before a ground state is reached are unnecessarily wasted. Schellnhuber et $a l^{15}$ suggested a Newton's method which searches directly for the solutions of $f_{i}=0$. The superconvergence of Newton's method greatly reduces the computing time. Since many force functions are nonmonotonic, an extremely good guess of the correct initial condition is required to apply the method successfully. The initial condition (8) is not a good choice since very often it will converge to a physically unstable configuration for large $k$ values. Schellnhuber et al gave an optimal initial configuration for a cosine external potential such as the one we are 
studying:

$$
x_{i}(0)=[i p / q]
$$

where [] stands for the integral part. The basic idea of this method is to put the atoms initially in the valleys of the potential. The system will then be trapped with certainty in the ground state before reaching a non-minimizing periodic orbit.

We have used both the differential equation method and Newton's method to calculate some commensurate ground states. They gave the same results but the latter method is much faster. When calculating a phase diagram or critical value $k_{c}$, we need to calculate a large number of ground states (a typical number for a phase diagram is 5000 ) or some high-period orbits (up to $q=17711$ ). The differential method is very difficult to apply in this case because it is extremely time-consuming.

\section{B. Phase Diagram}

The phase diagram consists of commensurate and incommensurate ground states in the parameter space of $k$ and $\mu$. For any given rational winding number $\omega$, there is a corresponding commensurate area (Arnold's tongue) in which $\omega$ is constant. Between any two tongues there is a gap which contains incommensurate states as well as higher order commensurate states.

The Farey tree construction ${ }^{16,17}$ can be used to study the phase diagram from low to high orders. There are $2^{n-1}+1$ rationals (hence tongues) in the n.th Farey generation in the interval $[0,1]$. The most effective way to construct a phase diagram is to locate the boundaries of commensurate states. For a given commensurate state $\omega=p / q$, its left boundary is determined by equating the 
energy of that tongue to the energy of an incommensurate state $\bar{\omega}$ in the immediate left neighborhood of the tongue. Since it has the same $k, \mu$ and is infinitely close to $\omega$, its energy should also be infinitely close. In practical calculations, the incommensurate $\bar{\omega}$ is approximated by a left neighboring tongue $\bar{\omega}=\bar{p} / \bar{q}$ of a much higher order, i.e., $\bar{q} \gg q$. The higher the order the better the approximation. We found that using $\bar{\omega}$ six orders higher than $\omega$ gives very good accuracy. The right boundary is determined in a similar way.

We divide the energy (5) into two parts: ${ }^{14}$ the lock-in energy $E_{\text {lock }}$ and the elastic energy $E_{\mathrm{el}}$ :

$$
\begin{aligned}
\mathcal{H} & =\sum_{i}\left[\frac{1}{\beta^{2}} e^{-\beta\left(x_{i+1}-x_{i}\right)}+\frac{k}{(2 \pi)^{2}}\left(1-\cos 2 \pi x_{i}\right)\right] \\
& +\sum_{i}\left[\frac{e^{-\beta \mu}}{\beta}\left(x_{i+1}-x_{i}-\mu\right)-\frac{1}{\beta^{2}} e^{-\beta \mu}\right] \\
\equiv & \mathcal{H}_{\text {lock }}(\beta ; \omega, k)+\mathcal{H}_{\mathrm{el}}(\beta ; \omega, \mu),
\end{aligned}
$$

where

$$
\mathcal{H}_{\text {lock }}(\beta ; \omega, k)=\sum_{i}\left[\frac{1}{\beta^{2}} e^{-\beta\left(x_{i+1}-x_{i}\right)}+\frac{k}{(2 \pi)^{2}}\left(1-\cos 2 \pi x_{i}\right)\right],
$$

and

$$
\mathcal{H}_{\mathrm{el}}(\beta ; \omega, \mu)=\frac{N}{\beta^{2}} e^{-\beta \mu}[\beta(\omega-\mu)-1]
$$

The $N$ in Eq. (18) is the total number of atoms in the system. The lock-in energy $\mathcal{H}_{\text {lock }}$ depends on the local atomic configurations $\left\{x_{i}\right\}$, and on $\omega$ and $k$, but not on the parameter $\mu$. The elastic energy $\mathcal{H}_{\mathrm{el}}$, on the other hand, does not depend on the local properties but only on the global properties of the system, $\omega$ and $\mu$. 
A boundary $\mu_{B}$ of a commensurate $\omega=p / q$ at a given $k$ is determined by the equation

$$
\mathcal{H}\left(\beta ; \omega, k, \mu_{B}\right)=\mathcal{H}\left(\beta ; \bar{\omega}, k, \mu_{B}\right)
$$

where $\bar{\omega}=\bar{p} / \bar{q}$ is the winding number of the neighboring tongue of a higher order. Using Eqs. (16)-(18), we can rewrite Eq. (19) to express $\mu_{B}$ explicitly:

$$
\mu_{B}=\frac{1}{\beta} \ln \left[\frac{\bar{\omega}-\omega}{\beta\left[h_{\mathrm{lock}}(\beta ; \omega, k)-h_{\mathrm{lock}}(\beta ; \bar{\omega}, k)\right]}\right],
$$

where $h_{\text {lock }}(\beta ; \omega, k)$ is the average lock-in energy per atom

$$
\begin{aligned}
h_{\mathrm{lock}}(\beta ; \omega, k) & =\frac{1}{N} \mathcal{H}_{\mathrm{lock}}(\beta ; \omega, k) \\
& =\frac{1}{q} \sum_{i=1}^{q}\left[\frac{1}{\beta^{2}} e^{-\beta\left(x_{i+1}-x_{i}\right)}+\frac{k}{(2 \pi)^{2}}\left(1-\cos 2 \pi x_{i}\right)\right] .
\end{aligned}
$$

We have used the expressions (20) and (21) to calculate the phase diagrams of the model (5) for different $\beta$ values (Fig. 2). Figure 2(a) is the phase diagram at $\beta=0.002$, which is used as a test of the $\beta \rightarrow 0$ limit. We can see that this phase diagram is almost the same as that of the standard FK model. It is symmetric about $\mu=\frac{1}{2}$. When $\beta$ is non-zero, the phase diagram becomes asymmetric [Figs. 2(b) and (c)], reflecting the nonlinear nature of the Toda interaction. The tongues all swing to the left. The tongnes with $\mu>\frac{1}{2}$ are bent more than the tongues with $\mu<\frac{1}{2}$, and their areas are also reduced. The right-most tongne, $\omega=1 / 1$, becomes larger. So in our model, the small $\mu$ part of the phase diagram remains a standard FK type, while in the large $\mu$ part of the diagram, the $\mu$ values are smaller compared to the standard FK model for the same stable commensurate states. As a result, the unity misfit occupies a large area in the phase diagram. 
For a system in which $\mu$ represents the pressure ${ }^{2,18}$, if the interatomic interaction is of the Toda type, then the commensurate states will occur at a lower pressure than those whose interaction is of the Hooke type. As $\beta$ increases the interaction weakens and this effect becomes greater [Fig. 1 and Figs. 2(b) and (c)].

\section{DEVIL'S STAIRCASE, $f(\alpha)$ AND $D_{q}$}

It has been shown ${ }^{18,19}$ that on or above the critical value $k_{c}$, the frequency ratio $\omega$ as a function of the parameter $\mu$ forms a complete Devil's staircase (DS) as shown in Fig. 3. This function contains only steps, each of them representing a stable commensurate state. Magnification of any part of the curve (not within a step) will reproduce the original curve. We study the Devil's staircase at the critical golden mean value $k_{c}\left(\omega_{G}\right)$. The Devil's staircase in Fig. 3 shows the influence of the Toda interaction: compared to the staircase of the standard FK model, the steps move to the left and get narrower as $\beta$ increases. The only exception is the $1 / 1$ step, which gets wider. The complementary set of a complete Devil's staircase, viz., the gaps between steps, is a fractal with zero measure. By defining a fractal measure on the fractal, we can study its multifractal properties. Consider a complete Devil's staircase of the $n$th Farey generation. The corresponding complementary set of this staircase has $2^{n-1}$ pieces (gaps) in the interval $[0,1]$. Denote by $\epsilon_{i}$ the width of the $i$ th piece, and $m_{i}$ the fractal measure ${ }^{14}$ defined to be the difference of the winding numbers of two neighboring steps:

$$
m_{i}=\omega_{i+1}-\omega_{i}, \quad i=1,2, \cdots, 2^{n-1}
$$


Thus $m_{i}$ satisfies the normalization condition

$$
\sum_{i} m_{i}=1
$$

The partition function ${ }^{20}$ of this multifractal in the $n$th Farey generation is then

$$
\Gamma^{(n)}(q, \tau)=\sum_{i=1}^{2^{n-1}} \frac{m_{i}^{q}}{\epsilon_{i}^{\tau}} .
$$

The function $\tau(q)$ can be obtained by equating (24) up to a finite constant $C$. We choose $C=1$. Then $\tau(q)$ is defined by

$$
\sum_{i=1}^{2^{n-1}} \frac{m_{i}^{q}}{\epsilon_{i}^{\tau(q)}}=1 .
$$

$\alpha(q)$ is defined to be the derivative of $\tau(q)$,

$$
\alpha(q)=\frac{d}{d q} \tau(q)
$$

Hance

$$
\alpha(q)=\frac{\sum_{i} \frac{m_{i}^{q}}{\epsilon_{i}^{\tau}} \ln m_{i}}{\sum_{i} \frac{m_{i}^{q}}{\epsilon_{i}^{\tau}} \ln \epsilon_{i}} .
$$

The singularity spectrum $f(\alpha)$ and the generalized dimension $D_{q}$ can then be calculated:

$$
\begin{aligned}
f(\alpha) & =q \alpha(q)-\tau(q), \\
D_{q} & =\frac{\tau(q)}{q-1} .
\end{aligned}
$$

We have calculated $f(\alpha)$ and $D_{q}$ of the Devil's staircase for different $\beta$ values. The numerical results are shown in Figs. 4 and 5. The singularity spectrum $f(\alpha)$ 
shows its dependence on the parameter $\beta$. It becomes ligher as $\beta$ increases. This is due to the fact that the Devil's steps decrease as $\beta$ increases, causing the fractal dimension of the fractal support to increase. The endpoints of the curve, $\alpha_{\min }$ and $\alpha_{\max }$ (corresponding to $q \rightarrow+\infty$ and $q \rightarrow-\infty$ respectively) also expand as $\beta$ increases: $\alpha_{\min }$ smaller and $\alpha_{\max }$ larger. The peak of the curve, $\alpha_{0}$, shifts to the right as $\beta$ increases, making the large- $\alpha$ side of the curve steeper. In the limit $\beta \rightarrow \infty, f(\alpha)$ will have a sawtooth shape, $\alpha_{0}=\alpha_{\text {max }}$. The change of the generalized dimension $D_{q}$ with parameter $\beta$ is shown in Fig. 5. A larger $\beta$ gives a $D_{q}$ curve with a higher kink.

\section{CRITICAL BEHAVIOR}

\section{A. Map and Critical Point}

The equilibrium configuration of the Hamiltonian (5) can be expressed as a map. The equilibrium configuration of the atoms is achieved by the zero force condition:

$$
\frac{\partial \mathcal{H}}{\partial x_{i}}=0
$$

Define the conjugate variable $y_{i}$,

$$
y_{i}=e^{-\beta\left(x_{i}-x_{i-1}\right)}
$$

Then condition (30) can be written in terms of a map:

$$
\left\{\begin{array}{l}
y_{i+1}=y_{i}-\frac{\beta k}{2 \pi} \sin 2 \pi x_{i}, \\
x_{i+1}=x_{i}-\frac{1}{\beta} \ln y_{i+1} .
\end{array}\right.
$$


When $k$ is small, there are KAM curves in the $(x, y)$ phase space. The last rotational KAM curve corresponding to the golden mean winding number breaks up at $k=k_{c}$, and the analytic invariant KAM curve becomes a Cantorus.

To study the critical behavior, we first have to locate the critical point $k_{c}$. We will use Greene's residue criterion ${ }^{21,22}$ to determine the critical point. Denote by $R_{i}^{e}(k)$ and $R_{i}^{h}(k)$ the residues of the elliptic and hyperbolic orbits with period $q_{i}$. The rational approximation of the golden mean winding number is given by the convergents $p_{i} / q_{i}$

$$
\omega_{\mathrm{G}}=\lim _{i \rightarrow \infty} \frac{p_{i}}{q_{i}}=\lim _{i \rightarrow \infty} \frac{F_{i}}{F_{i+1}}
$$

where the numbers $F_{i}$ define a Fibionacci sequence $F_{i+1}=F_{i}+F_{i-1}$ with $F_{0}=1$ and $F_{1}=1$. The residue criterion states

$$
\begin{aligned}
& \lim _{i \rightarrow \infty} R_{i}^{\epsilon}(k)= \begin{cases}0^{+} & k<k_{c} \\
a & k=k_{c} \\
\infty & k>k_{c}\end{cases} \\
& \lim _{i \rightarrow \infty} R_{i}^{h}(k)= \begin{cases}0^{-} & k<k_{c} \\
-b & k=k_{c} \\
-\infty & k>k_{c}\end{cases}
\end{aligned}
$$

where $a$ and $b$ are positive constants less than unity. The residue $R$ is defined via the Jacobian matrix $M$ of the linearized map (32):

$$
\left(\begin{array}{l}
\delta y_{i+1} \\
\delta x_{i+1}
\end{array}\right)=M_{i}\left(\begin{array}{l}
\delta y_{i} \\
\delta x_{i}
\end{array}\right)
$$

where

$$
M_{i}=\left(\begin{array}{cc}
1 & -\beta k \cos 2 \pi x_{i} \\
-\frac{1}{\beta y_{i+1}} & 1+\frac{k \cos 2 \pi x_{i}}{y_{i+1}}
\end{array}\right) .
$$

For a $q$-orbit, $M$ is given by

$$
M=\prod_{i=1}^{q} M_{i}
$$


The residue $R$ of that orbit is defined $b y^{21}$

$$
R=\frac{1}{4}(2-\operatorname{Tr} M)
$$

To calculate $k_{c}$, we use the map combined with Newton's method to reduce the computing time. The symmetry lines greatly simplify the searching for periodic orbits in the $(x, y)$ space. The map (32) has four symmetry lines:

$$
\left\{\begin{array}{l}
x=0 \\
x=\frac{1}{2} \\
x=-\frac{1}{2 \beta} \ln y \\
x=\frac{1}{2}-\frac{1}{2 \beta} \ln y .
\end{array}\right.
$$

It has been observed ${ }^{21,23}$ that for a $q$-orbit there are at least two out of its $q$ points which lie on the symmetry lines. The search for a $q$-orbit is then limited to these four lines instead of the whole $(x, y)$ space.

We have calculated the $k_{c}$ values of the golden mean winding number $\omega_{c}$ for different $\beta$ values. Table I lists the numerical values of $k_{c} \cdot{ }^{24}$ Also listed are $k_{c}\left(\omega_{\bar{c}}\right)$ where $\omega_{G}=1-\omega_{G}$ for some selected $\beta$ values. At $\beta=0$, which is the standard FK model, $k_{c}\left(\omega_{G}\right)=k_{c}\left(\omega_{G}\right)$. But $k_{c}\left(\omega_{G}\right)$ and $k_{c}\left(\omega_{G}\right)$ become distinct as $\beta \neq 0$, and their difference increases as $\beta$ increases. This reflects the asymmetry of the map (32). For the standard FK model, its map, the standard map,

$$
\begin{aligned}
& y_{i+1}=y_{i}+\frac{k}{2 \pi} \sin 2 \pi x_{i}, \\
& x_{i+1}=x_{i}+y_{i+1}
\end{aligned}
$$

is invariant under the interchange of $\omega$ and $1-\omega$, i.e., $y \rightarrow 1-y$. The map (32) of the Toda interaction does not have this symmetry due to the exponential form of 
the Toda interaction (logarithm term in the map). As a result, the $k_{c}$ values for $\omega_{G}$ and $\omega_{\bar{G}}$ become asymmetric. However, we will see in the next section that the critical exponents at $k_{c}\left(\omega_{G}\right)$ and $k_{c}\left(\omega_{G}\right)$ are identical.

\section{B. Critical Exponents}

At the critical point $k=k_{c}$, there is a transition by breaking of analyticity ${ }^{11}$. The hull function describing the incommensurate structure undergoes a transition from an analytic function to a discontinuous function. Many physical quantities ${ }^{11}$ also undergo a transition at $k_{c}$. We will study their critical behavior in our model.

First consider the gap in the phonon spectrum $\Omega_{G}$. Consider a small viloration of atoms around their equilibrium positions $\left\{x_{i}\right\}$

$$
x_{i}(t)=x_{i}+\epsilon_{i}(t)
$$

The equation of motion for this vibration is described by

$$
\frac{d^{2} x_{i}(t)}{d t^{2}}=-\frac{\partial \mathcal{H}\left(\left\{x_{i}(t)\right\}\right)}{\partial x_{i}(t)}
$$

Since the equilibrium position $\left\{x_{i}\right\}$ is determined by the condition

$$
\left.\frac{\partial \mathcal{H}\left(\left\{x_{i}(t)\right\}\right)}{\partial x_{i}(t)}\right|_{x_{i}(t)=x_{i}}=0
$$

the linearized equation of motion of small vibrations is given by

$$
\delta \ddot{x}_{i}(t)+\sum_{j} \frac{\partial^{2} \mathcal{H}\left(\left\{x_{i}(t)\right\}\right)}{\partial x_{i}(t) \partial x_{j}(t)} \delta_{j}(t)=0, \quad i=1,2, \ldots, q
$$

where $\ddot{x}=d^{2} x / d t^{2}$ and

$$
\frac{\partial^{2} \mathcal{H}}{\partial x_{i} \partial x_{j}}= \begin{cases}-e^{-\beta\left(x_{i+1}-x_{i}\right)} & j=i+1 \\ e^{-\beta\left(x_{i+1}-x_{i}\right)}+e^{-\beta\left(x_{i}-x_{i-1}\right)}+k \cos 2 \pi x_{i} & j=i \\ -e^{-\beta\left(x_{i}-x_{i-1}\right)} . & j=i-1\end{cases}
$$


Performing a time Fourier trausform of Eq. (43) gives

$$
\begin{gathered}
-e^{-\beta\left(x_{i}-x_{i-1}\right)} \epsilon_{i-1}+\left[e^{-\beta\left(x_{i+1}-x_{i}\right)}+e^{-\beta\left(x_{i}-x_{i-1}\right)}+k \cos 2 \pi x_{i}-\Omega^{2}\right] \epsilon_{i} \\
-e^{-\beta\left(x_{i+1}-x_{i}\right)} \epsilon_{i+1}=0 . \quad i=1,2, \ldots, q
\end{gathered}
$$

Define two functions $D\left(x_{i+1}, x_{i}, x_{i-1}\right)$ and $N\left(x_{i}, x_{i-1}\right)$ :

$$
\begin{aligned}
D\left(x_{i+1}, x_{i}, x_{i-1}\right) & =e^{-\beta\left(x_{i+1}-x_{i}\right)}+e^{-\beta\left(x_{i}-x_{i-1}\right)}+k \cos 2 \pi x_{i}, \\
N\left(x_{i}, x_{i-1}\right) & =-e^{-\beta\left(x_{i}-x_{i-1}\right)} .
\end{aligned}
$$

Then the eigenvalue equation (45) can be written as

$$
\left(\begin{array}{ccccc}
D\left(x_{2}, x_{1}, x_{0}\right)-\Omega^{2} & N\left(x_{2}, x_{1}\right) & 0 & \ldots & N\left(x_{1}, x_{0}\right) \\
N\left(x_{2}, x_{1}\right) & D\left(x_{3}, x_{2}, x_{1}\right)-\Omega^{2} & N\left(x_{3}, x_{2}\right) & \ldots & 0 \\
0 & N\left(x_{3}, x_{2}\right) & D\left(x_{4}, x_{3}, x_{2}\right)-\Omega^{2} & \ldots & 0 \\
N\left(x_{q}, x_{q}\right) & & \vdots & & \\
& & 0 & \ldots & D\left(x_{q+1}, x_{q}, x_{q-1}\right)-\Omega^{2}
\end{array}\right)=0
$$

with the periodic boundary condition (13).

The phonon spectrum $\left\{\Omega_{i}\right\}$ is obtained by solving this $q \times q$ matrix equation. The gap in the phonon spectrum $\Omega_{G}$ is defined to be the lowest phonon frequency in the system, $\Omega_{G}=\min \left\{\Omega_{i}\right\}$. For $k<k_{c}$, the ground state of the chain is in a sliding mode and the atomic positions can be described by a hull function ${ }^{18} f$

$$
x_{i}=f(i \omega+\alpha)=i \omega+\alpha+g(i \omega+\alpha) \text {, }
$$

where $\alpha$ is an arbitrary phase. $g$ is a continuous periodic function with the same period as the external potential. Since the hull function is analytic for $k<k_{c}$. we can substitute Eq. (49) for $x_{i}$ in Eq. (42) and differentiate Eq. (42) with respect 
to the pliase $\alpha$

$$
\begin{gathered}
-e^{-\beta\left(x_{i}-x_{i-1}\right)} f_{i-1}^{\prime}+\left[e^{-\beta\left(x_{i+1}-x_{i}\right)}+e^{-\beta\left(x_{i}-x_{i-1}\right)}+k \cos 2 \pi x_{i}\right] f_{i}^{\prime} \\
-e^{-\beta\left(x_{i+1}-x_{i}\right)} f_{i+1}^{\prime}=0, \quad i=1,2, \ldots, q
\end{gathered}
$$

where $f_{i}^{\prime}=(\partial / \partial \alpha) f(i \omega+\alpha)$. The Fourier transform of $(50)$ shows that $f_{i}^{\prime}(\omega)$ is a solution of Eq. (45) with $\Omega=0$. Therefore $\Omega_{G}=0$ for $k<k_{c}$. As $k>k_{c}$, a gap $\Omega_{G}$ in the phonon spectrum appears and the critical behavior of $\Omega_{G}$ can be characterized by the exponent ${ }^{11} \chi$

$$
\Omega_{G}(k) \sim\left(k-k_{c}\right)^{\prime}
$$

We have calculated $\chi$ for various $\beta$ values, from $\beta=0.002$ up to $\beta=10$. Despite the fact that $k_{c}$ increases more than two orders in this $\beta$ range and the phase diagram changes greatly, $\chi$ remains unchanged. Our numerical estimate of $\chi$ is

$$
\chi=1.02 \pm 0.01
$$

The $\chi$ value for $\omega_{\tau}$ is found to be the same as that for $\omega_{G}$. We next study the critical behavior of the correlation length.

The correlation length $\xi$ measures the distance over which a perturbation $\delta x_{i}$ propagates along the chain. An infinitesimal displacement $\delta x_{i}$ at $x_{i}$ will cause a displacement $\delta x_{j}$ at $x_{j}$, where

$$
\delta x_{j} \sim \exp (-|j-i| / \xi) \delta x_{i}
$$

Eq. (53) defines the correlation length of the ground state. It can be shown ${ }^{11}$ that $\xi$ is the inverse of the Lyapunov exponent $\gamma$

$$
\xi=\frac{1}{\gamma} .
$$


$\xi$ and $\gamma$ thus share the same critical exponent $\nu$. We will use $\gamma$ to calculate the exponent $\%$. The Lyapunov exponent can be calculated from the eigenvalue of the Jacolbian matrix $M(37)$ :

$$
\gamma=\lim _{q \rightarrow \infty} \frac{1}{q} \ln \left|\lambda_{l}\right|
$$

where $\left|\lambda_{l}\right|$ is the modulus of the larger eigenvalue of $M$, and $q$ is the period of the orbit. The eigenvalues $\lambda$ of the matrix $M$ can be expressed in terms of its trace $\operatorname{Tr} M$

$$
\lambda_{ \pm}=\frac{1}{2} \operatorname{Tr} M\left[1 \pm \sqrt{1-\left(\frac{2}{\operatorname{Tr} M}\right)^{2}}\right] .
$$

In the sliding mode $k<k_{c}, \quad \xi \rightarrow \infty$ since the chain can slide freely under an infinitesimal displacing force. For $k>k_{c}$, the atoms are locked, $\xi$ therefore take a finite value and so does $\gamma$. The critical exponent $\nu$ describes the critical behavior of $\gamma$ :

$$
\gamma(k) \sim\left(k-k_{\mathrm{c}}\right)^{\nu}
$$

As in the case of $\chi$, the values of $\nu$ for various $\beta$ are all the same. We have calculated $\nu$ for $\beta$ from 0.002 to 10 , and no appreciable difference is found. Also the value of $\nu$ at $k_{c}\left(\omega_{\bar{G}}\right)$ is identical to that at $k_{c}\left(\omega_{G}\right)$ :

$$
\nu=0.99 \pm 0.01
$$

The Peierls-Nabarro (PN) barrier of the ground state is defined to be the minimal energy barrier that must be overcome to continuously translate the chain of atoms on the external potential. For $k<k_{c}$, the PN barrier $E_{P N}$ vanishes since no extra energy is needed to shift the chain in this sliding mode. For $k>k_{c}$, the ground state is described by a discontinuous hull function which in the $(x, y)$ phase 
space is represented by a Cantor set. A minimizing periodic orlbit $\left\{\left(y_{i}, x_{i}\right)\right\}_{i=1}^{q}$ can be used to approximate this Cantor $\operatorname{set}^{25}$. The PN barrier $E_{P N}$ is the energy difference between the minimizing orbit and its companion minimax orbit

$$
E_{P N}=E_{\max }(\omega)-E_{\min }(\omega)
$$

where $E_{\max }(\omega)\left[E_{\min }(\omega)\right]$ is the energy of the minimax (minimizing) orbit of winding number $\omega$. The critical behavior of $E_{P N}$ obeys the following power law

$$
E_{P N} \sim\left(k-k_{c}\right)^{\psi}
$$

The critical exponent $\psi$ is found to be

$$
\psi=3.00 \pm 0.02
$$

for all $\beta$ values we have calculated. $\psi$ is also the same for $k_{c}\left(\omega_{G}\right)$ and $k_{c}\left(\omega_{\bar{G}}\right)$. The scaling law observed in Ref. 11 also holds in the FK model with Toda interactions

$$
\psi=2 \chi+\nu
$$

Since all the critical exponents we have calculated are the same for the standard FK model and the FK model with Toda interactions; therefore these two models belong to the same universality class in the conventional sense.

\section{CONCLUSIONS AND DISCUSSIONS}

We have studied a strongly nonlinear system: the FK model with Toda interactions. With an adjustable nonlinearity parameter $\beta$, this model includes both the standard FK model $(\beta \rightarrow 0)$ and the hard-rod model $(\beta \rightarrow \infty)$. A number 
of new features appear due to the exponential form of the Toda interaction. The phase diagram becomes asymmetric when $\beta \neq 0$, which reflects the breaking of the reflectional symmetry observed in the standard FK model. This asymmetry increases with $\beta$. The singularity spectrum $f(\alpha)$ and the generalized dimension $D_{q}$ also show their dependence on $\beta$. In particular, the fractal dimension of the support increases as $\beta$ increases. At the hard-rod limit, $f(\alpha)$ has a sawtooth shape. The Toda interaction also destroys the symmetry of $\omega \rightarrow 1-\omega$ in the map of standard FK model. As a result, the critical $k_{c}$ values at $\omega_{G}$ and $\omega_{G}=1-\omega_{G}$ are distinct. Both of these two critical points are functions of $\beta$. However, the critical exponents of the gap in the phonon spectrum, the correlation length and the Peierls-Nabarro barrier, at $k_{c}\left(\omega_{G}\right)$ and $k_{c}\left(\omega_{G}\right)$ as well as different $\beta$ values, are all identical. This shows that the FK model with Toda interactions belongs to the same universality class as the standard FK model. This is in marked contrast to a nonanalytic FK model studied by Shi and $\mathrm{Hu}^{26}$

In our calculations of $f(\alpha)$ and the critical exponents, $k_{c}\left(\omega_{G}\right)$ was used as the critical points. While $k_{c}\left(\omega_{G}\right)$ is indeed the largest $k_{c}(\omega)$ in the standard FK model (corresponding to the standard map), it is not the case for Toda interactions. As we have seen, $k_{c}\left(\omega_{\bar{G}}\right)$ is greater than $k_{c}\left(\omega_{G}\right)$ for non-zero $\beta$, and the difference increases with $\beta$. Yet $k_{c}\left(\omega_{\vec{G}}\right)$ is still not necessarily the largest $k_{c}(\omega)$. In principle, one needs to calculate $k_{c}$ for every periodic $\omega$ involved to get a critical line $k_{c}\left(\omega_{i}\right)$. Using a single $k_{c}\left(\omega_{G}\right)$ to approximate the critical line may affect the Devil's staircase and therefore the $f(\alpha)$ spectrum. However, the critical exponents do not seem to be affected by this approximation. 


\section{Acknowledgements}

This work was supported in part by .... This work was completed while one of us $(\mathrm{BH})$ was visiting the Institute of Physics of Academia Sinica in the summer of 1991. He would like to thank Dr. C.K. Hu for the invitation and the National Science Council for support. BL would like to thank Dr. P.H. Hor for summer support. 


\section{REFERENCES}

1. Y.I. Frenkel and T. Kontorova, Zh. Eksp. Teor. Fiz. 8, 1340 (1938).

2. P. Bak, Rep. Prog. Phys. 45. 587 (1982).

3. G. Grumer, Commun. Solid State Phys. 10, 183 (1983).

4. I.E. Dzyaloshinskii, Zh. Eksp. Teor. Fiz. 46, 1420 (1964); Sov. Phys. JETP 23, 960 (1964).

5. M.S. Dresselhaus and G. Dresselhaus, Adv. Phys. 30, 139 (1981).

6. D. Schechtman, I. Blech, D. Gratios and J.W. Cahn, Phys. Rev. Lett. 53, 1951 (1984).

7. F. Nabarro, "Theory of Crystal Dislocation", Clarendon, Oxford, 1967.

8. S.C. Ying, Phys. Rev. B 3, 4160 (1971).

9. M. Toda, J. Phys. Soc. Jpn. 22, 431 (1967).

10. A. Milchev, Phys. Rev. B 33, 2062 (1986);

A. Milchev and G.M. Mazzucchelli, Phys. Rev. B 38, 2808 (1988)

11. M. Peyrard and S. Aubry, J. Phys. C 16, 1593 (1983).

S. Aubry, in Proceedings of the Les Houches Lectures, ed. R. Balian and R. Stora, North-Holland, Amsterdam, 1981.

12. S. Aubry, Physica 7D, 240 (1983).

13. S. Aubry and P.Y. Le Daeron, Physica 8D, 381 (1983).

14. O. Biham and D. Mukamel. Phys. Rev. A 39,5326 (1989).

15. H.J. Schellnhuber, H. Urbschat and A. Block, Phys. Rev. A 33, 2850 (1986).

16. I. Niven, "Diophantine Approximations", Wiley, New York, 1960. 
17. I. Niven and H.S. Zuckerman, "An Introduction to the Theory of Numbers, Wiley, New York, 1980.

18. S. Aubry, in "Solitons and Condensed Matter Physics", ed. A.R. Bishop and T. Schneider, Springer-Verlag, Berlin, 1979.

19. L. de Seze and S. Aubry, J. Phys. C 17, 389 (1984).

20. T.C. Halsey, M.H. Jensen, L.P. Kadanoff, I. Procaccia and B.I. Shraiman, Phys. Rev. A 33, 1141 (1986).

21. J.M. Greene, J. Math. Phys. 20, 1183 (1979).

22. S.J. Shenker and L.P. Kadanoff, J. Stat. Phys. 27, 631 (1982).

23. J.M. Greene, R.S. Mackay, F. Vivaldi and M.J. Feigenbaum, Physica 3D, $468(1981)$.

24. Some of the $k_{c}$ values are provided by J. Shi.

25. H. Johannesson, B. Schaub and H. Suhl, Phys. Rev. B 37, 9625 (1988).

26. J. Shi and B. Hu (to be published). 


\section{Figure Captions}

Fig. 5.1 The Toda interaction $W(r)$ as a function of $r=x_{i+1}-x_{i}$ at the parameter $\mu=0.5$

Fig. 5.2 The $W_{0}(r)$ and $W(r)$ at the limit $\beta \rightarrow \infty$. (a) $W_{0}(r),(\mathrm{b}) W(r)$.

Fig. 5.3 Phase diagram of the FIK model with Toda interactions at the 5th Farey generation. (a) $\beta=0.002$, the standard FK model, (b) $\beta=2.0$, the phase diagram becomes anymmetry, (c) $\beta=5.0$, the effect of Toda interaction is greater.

Fig. 5.4 Complete Devil's staircases of the FK model with Toda interactions.

The staircases of the 5 th Farey generation are shown. (a) $\beta=0.002$, the standard FK model, (b) $\beta=2.0$.

Fig. 5.5 Singularity spectrum $f(\alpha)$ for the multifractals of complete Devil's staircases for various $\beta$ values.

Fig. 5.6 Generalized dimension $D_{q}$ for the same multifractal as in figure 5.5. 


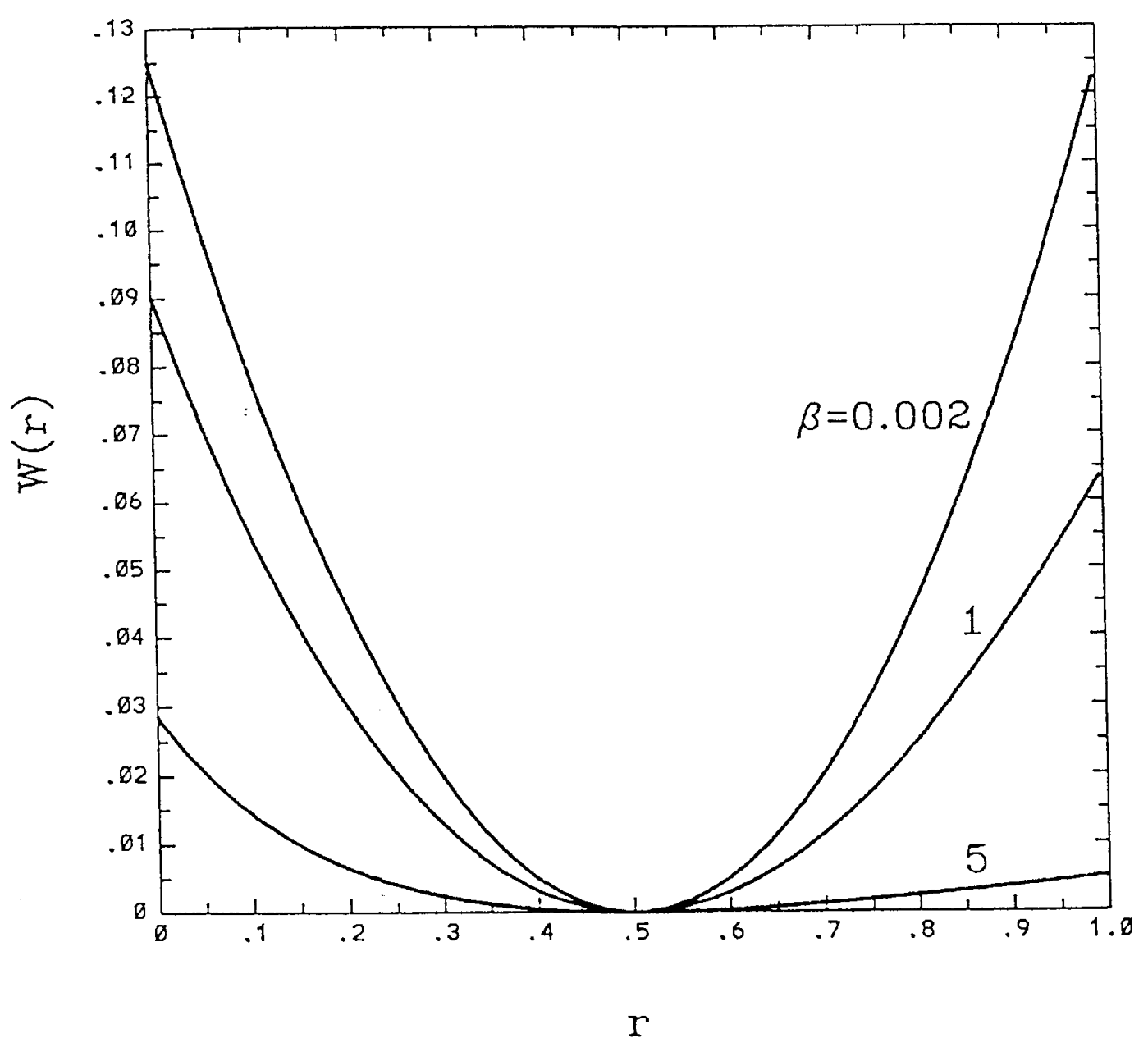

Fig. 5.1 


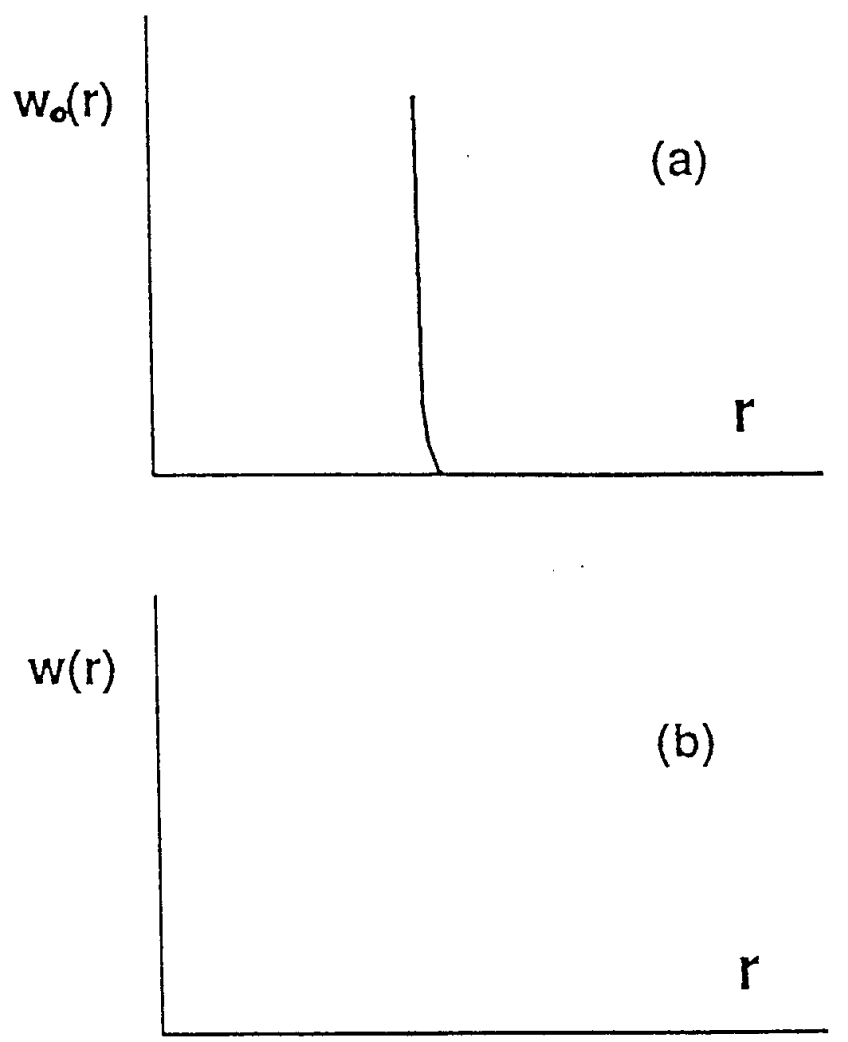

Fig. 5.2 
3

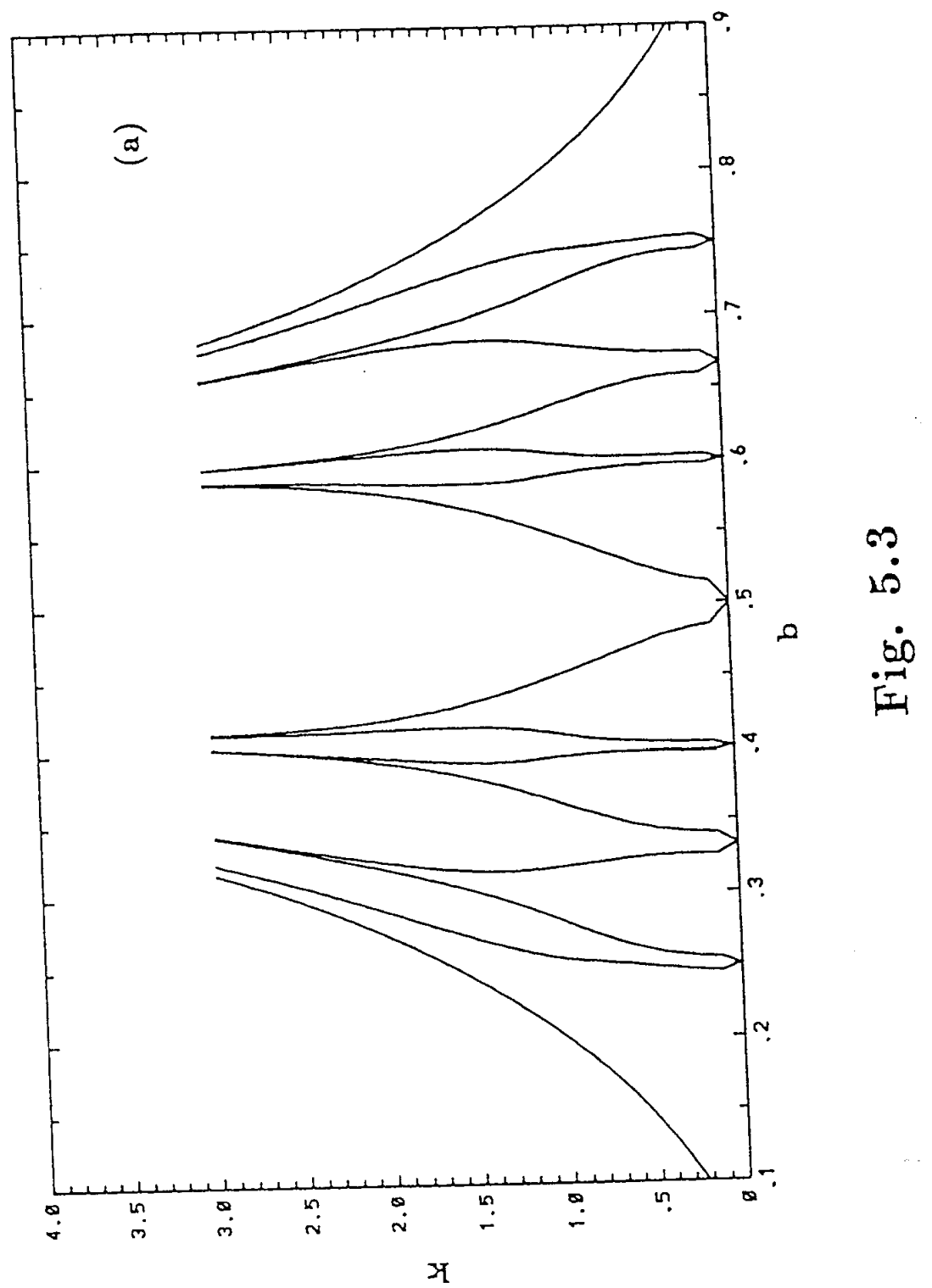




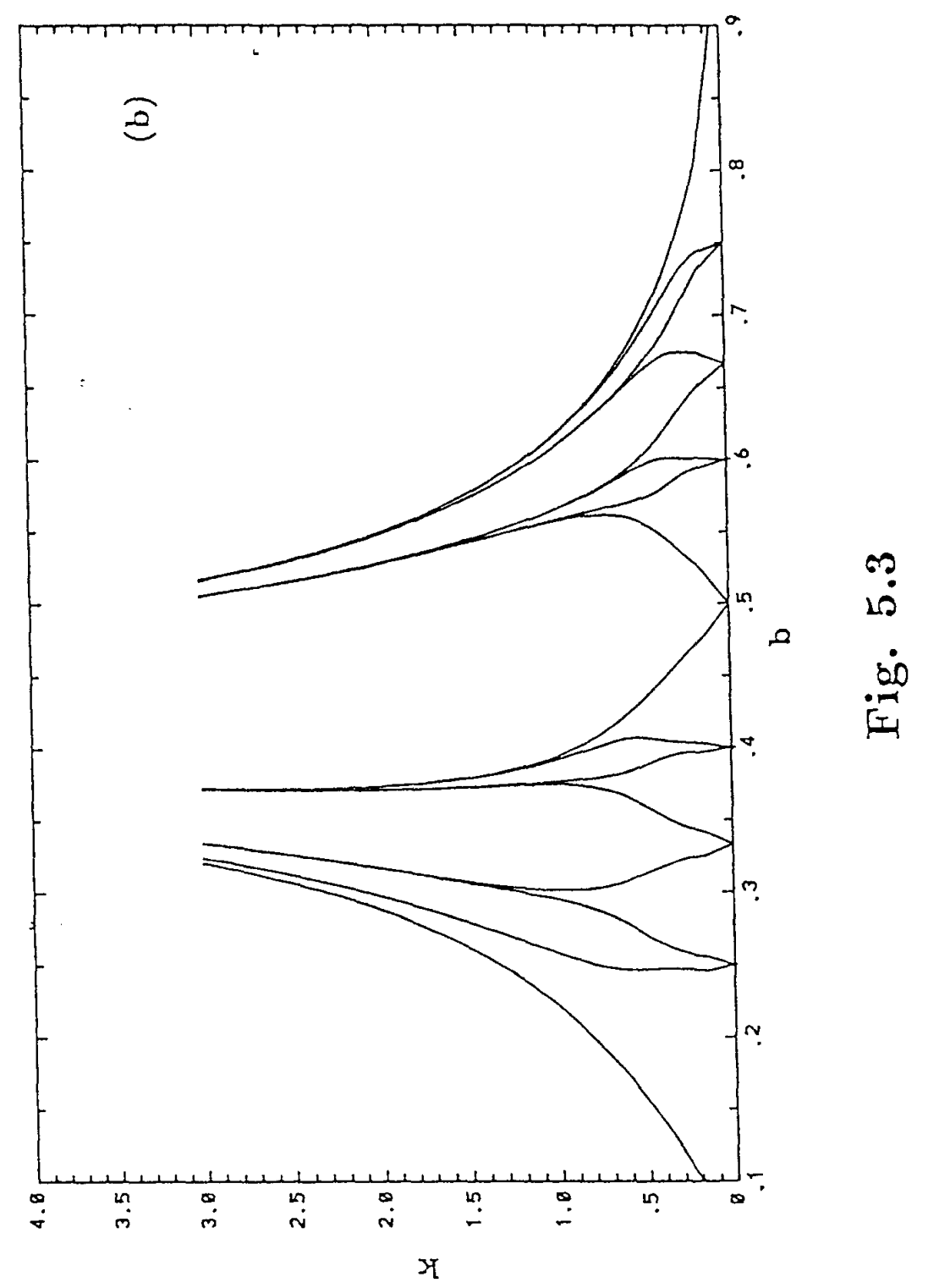




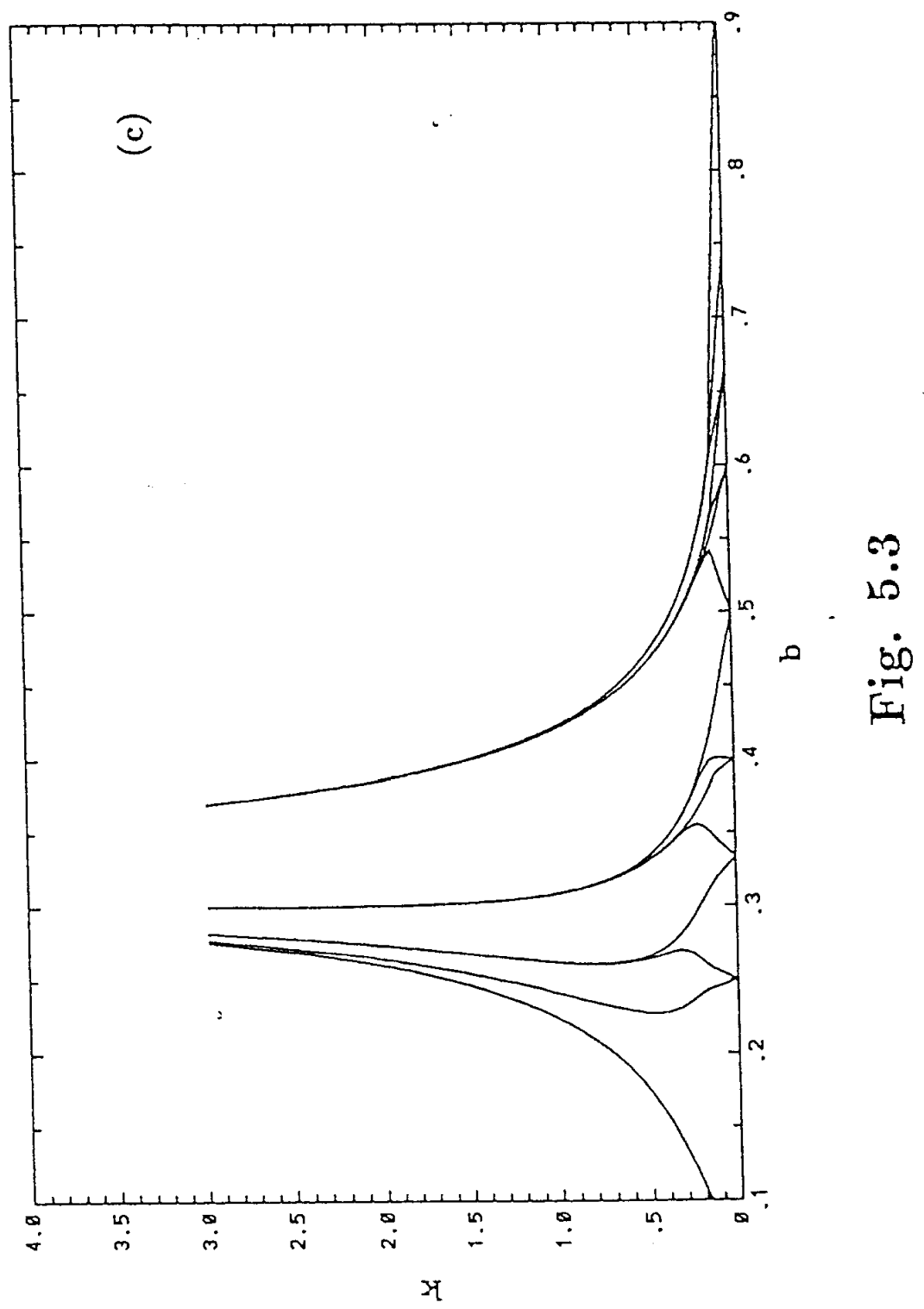




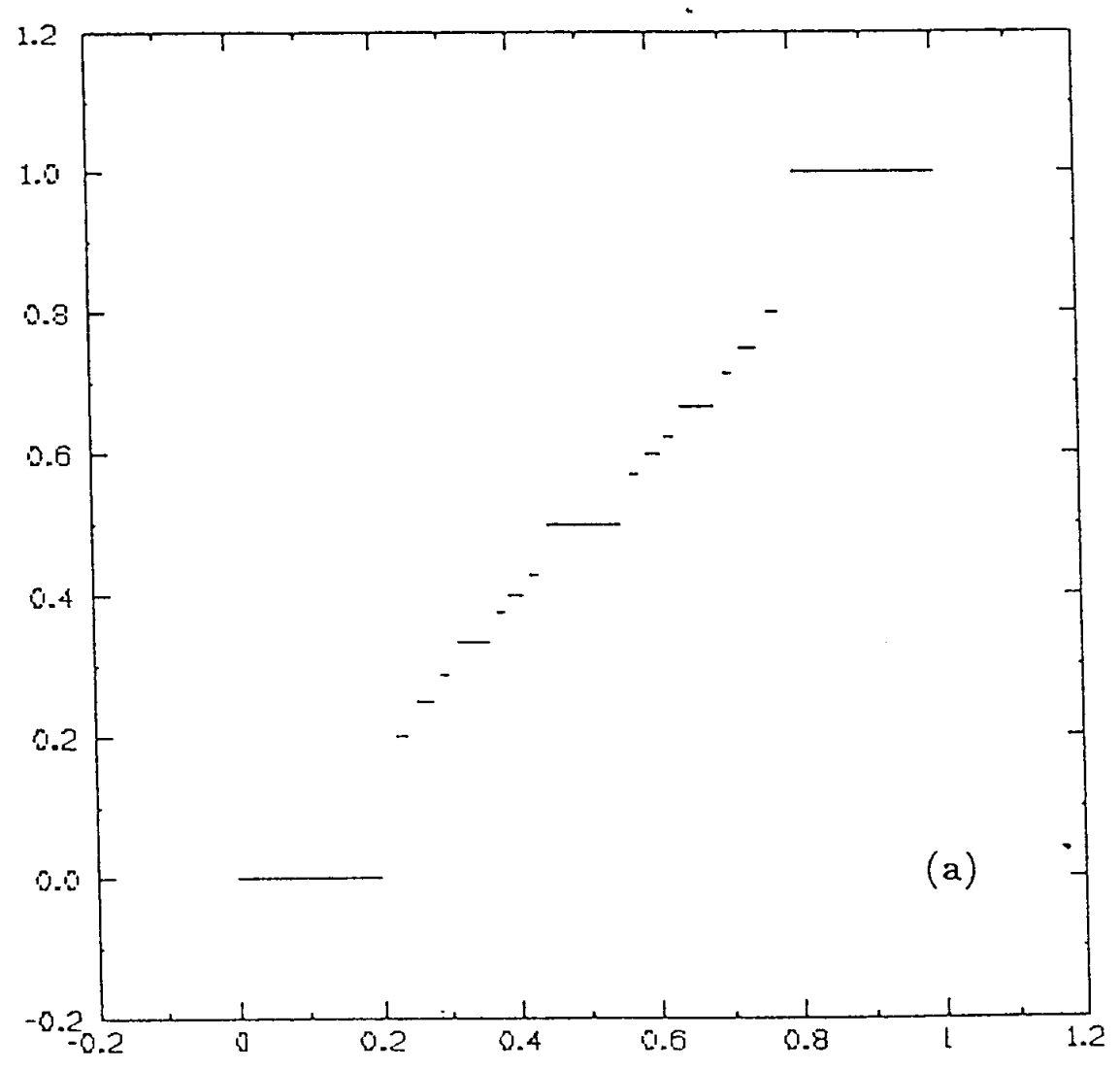

Fig. 5.4 
3

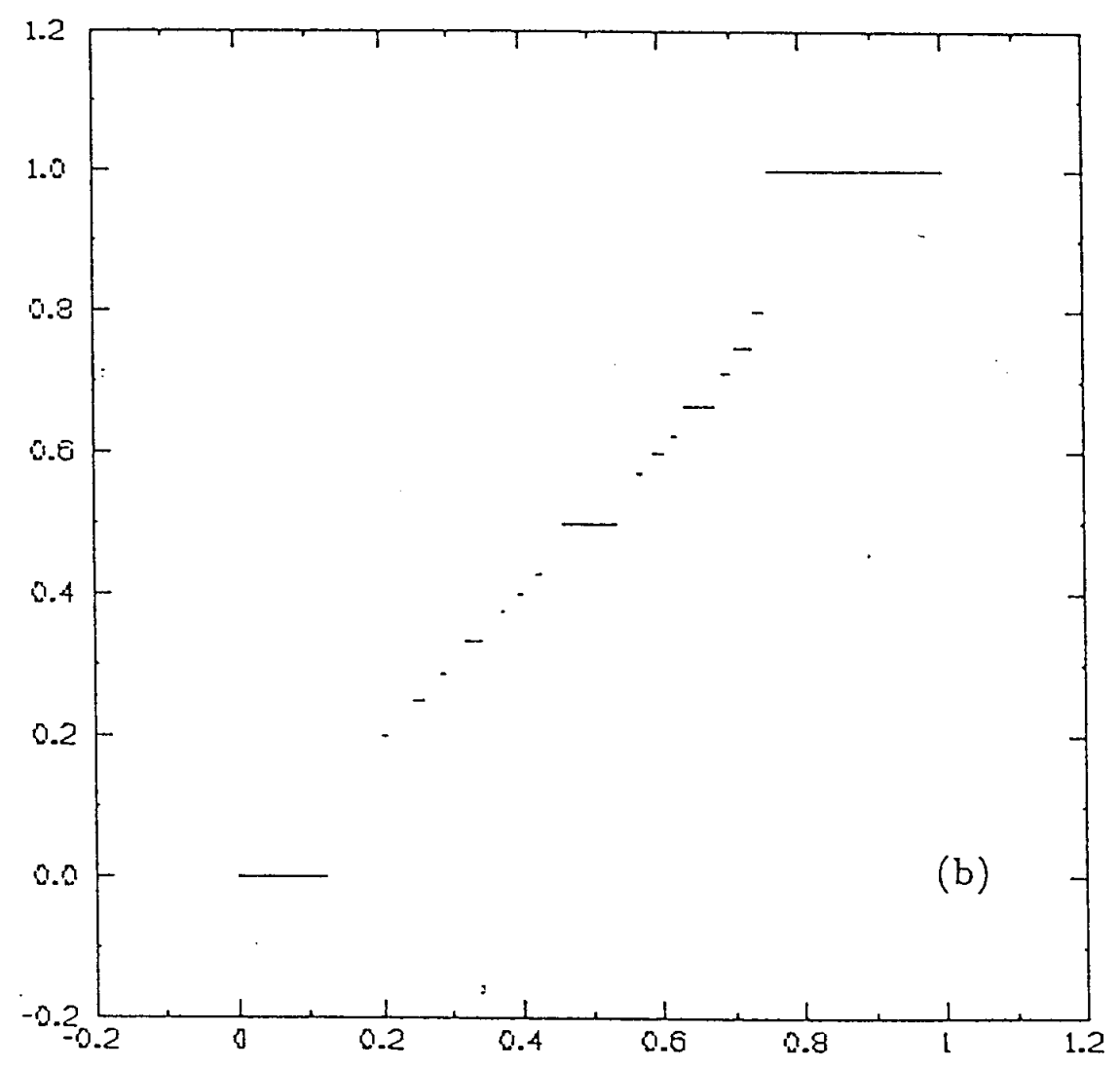

Fig. 5.4

$j$ 


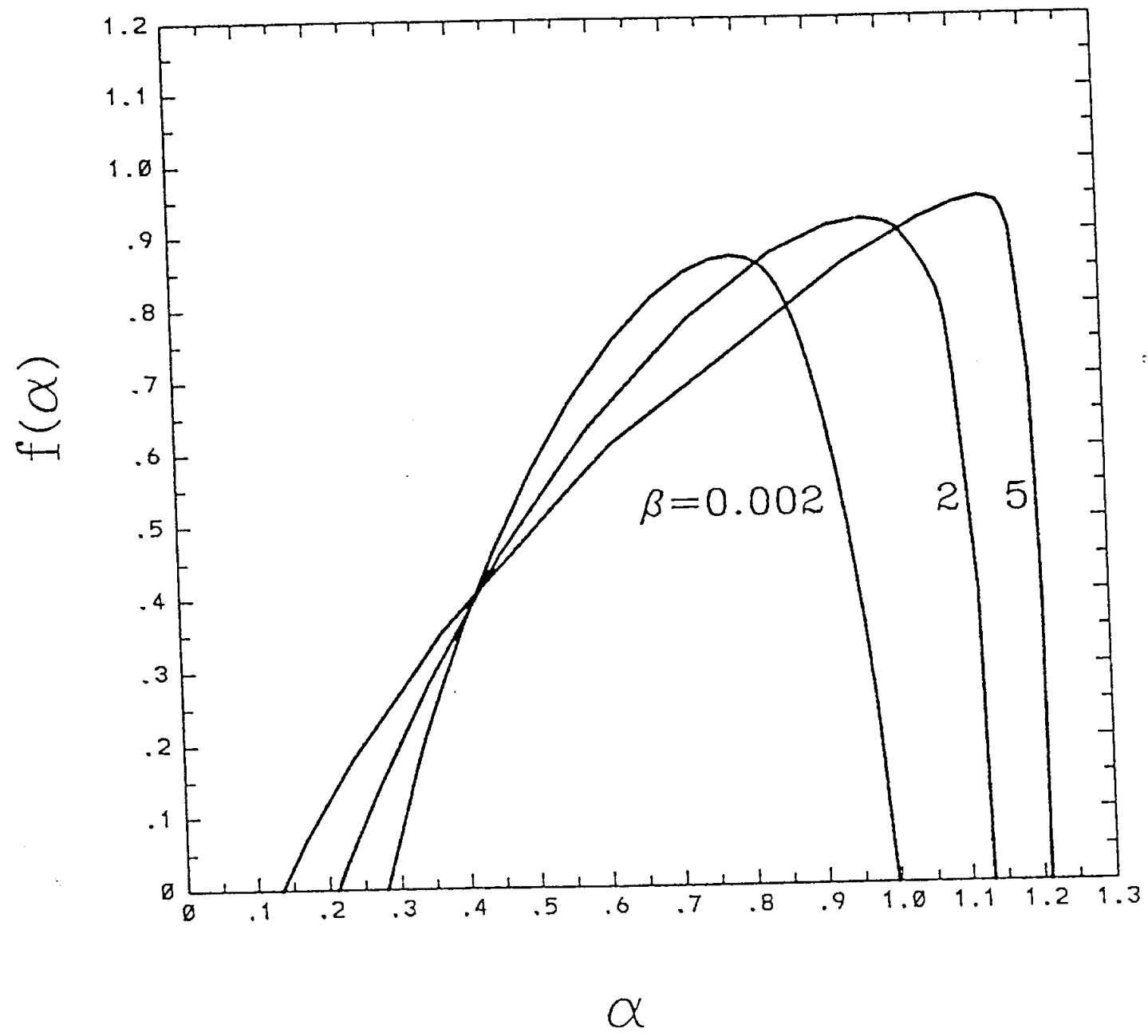

Fig. 5.5 


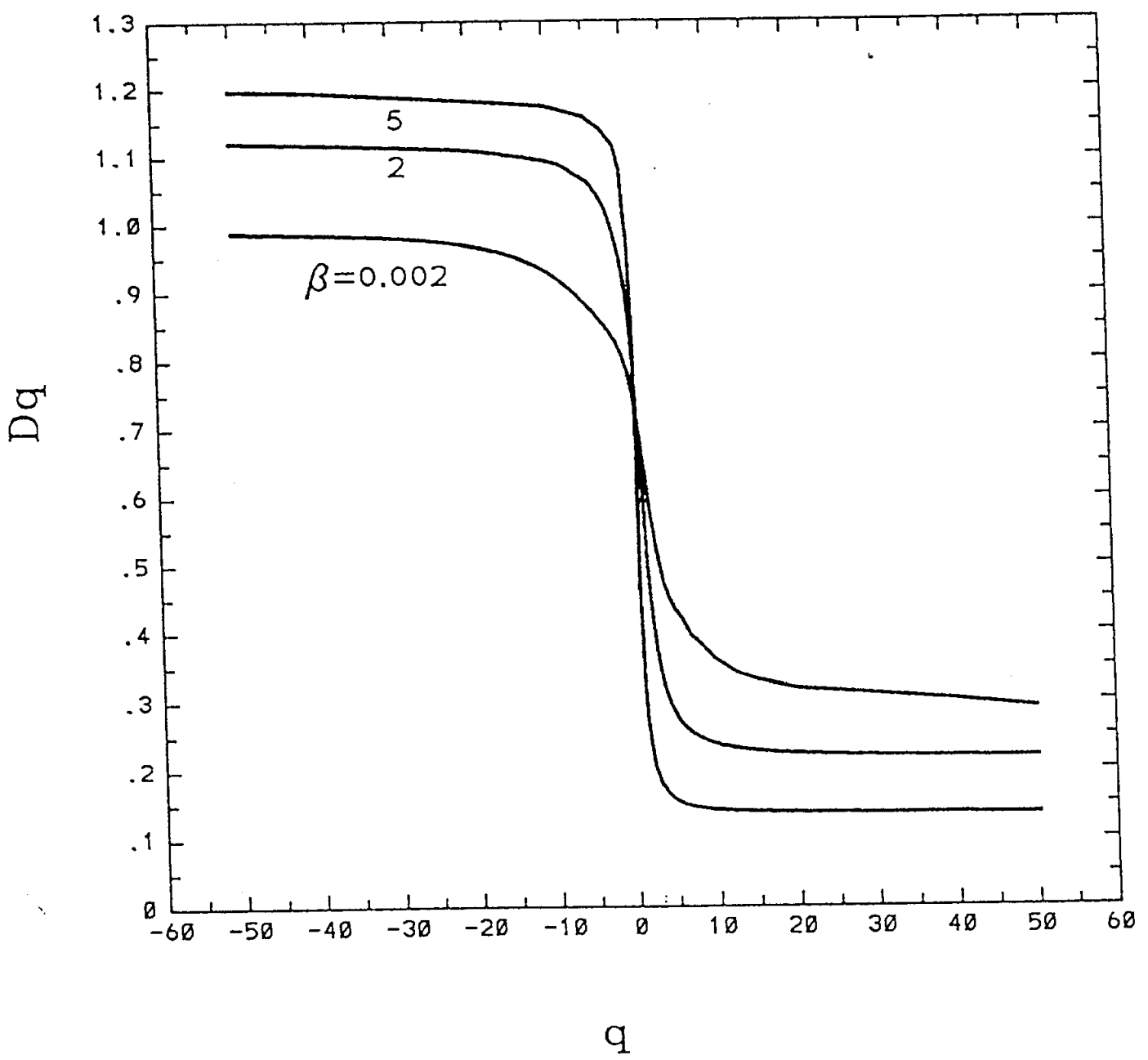

Fig. 5.6

2 
Table I Critical point $k_{c}$ of the winding numbers $\omega_{G}$ and $\omega_{G}$ for various $\beta$.

\begin{tabular}{|c|c|c|}
\hline$\beta$ & $k_{c}\left(\omega_{G}\right)$ & $k_{c}\left(\omega_{\sigma}\right)$ \\
\hline 0.0 & 0.971635 & 0.971635 \\
\hline 0.002 & 0.970473 & 0.970842 \\
\hline 0.1 & 0.915184 & 0.933420 \\
\hline 1.0 & 0.534561 & \\
\hline 2.0 & 0.294788 & 0.437262 \\
\hline 5.0 & 0.0502393 & \\
\hline 10.0 & 0.00284025 & \\
\hline
\end{tabular}

